\title{
Application of Machine Learning Techniques to Identify Data Reliability and Factors Affecting Outcome After Stroke Using Electronic Administrative Records
}

\author{
Santu Rana ${ }^{1}$, Wei Luo ${ }^{2}$, Truyen Tran ${ }^{1}$, Svetha Venkatesh ${ }^{1}$, Paul Talman ${ }^{3}$, Thanh Phan ${ }^{4}$, \\ Dinh Phung ${ }^{5}$ and Benjamin Clissold ${ }^{3,4,6 *}$ \\ ${ }^{1}$ Applied Artificial Intelligence Institute (A2I2), Deakin University, Geelong, VIC, Australia, ${ }^{2}$ School of Information Technology, \\ Deakin University, Burwood, VIC, Australia, ${ }^{3}$ Neurosciences Department, University Hospital Geelong, Geelong, VIC, \\ Australia, ${ }^{4}$ Stroke and Ageing Research Group, Department of Medicine, Monash University, Melbourne, VIC, Australia, \\ ${ }^{5}$ Department of Science and Al, Monash University, Clayton, VIC, Australia, ${ }^{6}$ Department of Epidemiology and Preventive \\ Medicine, Monash University, Melbourne, VIC, Australia
}

\section{OPEN ACCESS}

Edited by:

David S. Liebeskind, University of California, Los Angeles,

United States

Reviewed by:

Steffen Tiedt,

LMU Munich University

Hospital, Germany

Sofia Vallila Rohter, MGH Institute of Health Professions, United States

*Correspondence:

Benjamin Clissold Benjamin.Clissold1@monash.edu

Specialty section:

This article was submitted to Stroke,

a section of the journa

Frontiers in Neurology

Received: 21 February 2021

Accepted: 30 August 2021

Published: 27 September 2021

Citation:

Rana S, Luo W, Tran T, Venkatesh S,

Talman P, Phan T, Phung D and Clissold B (2021) Application of

Machine Learning Techniques to Identify Data Reliability and Factors Affecting Outcome After Stroke Using

Electronic Administrative Records.

Front. Neurol. 12:670379

doi: 10.3389/fneur.2021.670379
Aim: To use available electronic administrative records to identify data reliability, predict discharge destination, and identify risk factors associated with specific outcomes following hospital admission with stroke, compared to stroke specific clinical factors, using machine learning techniques.

Method: The study included 2,531 patients having at least one admission with a confirmed diagnosis of stroke, collected from a regional hospital in Australia within 2009-2013. Using machine learning (penalized regression with Lasso) techniques, patients having their index admission between June 2009 and July 2012 were used to derive predictive models, and patients having their index admission between July 2012 and June 2013 were used for validation. Three different stroke types [intracerebral hemorrhage $(\mathrm{ICH})$, ischemic stroke, transient ischemic attack (TIA)] were considered and five different comparison outcome settings were considered. Our electronic administrative record based predictive model was compared with a predictive model composed of "baseline" clinical features, more specific for stroke, such as age, gender, smoking habits, co-morbidities (high cholesterol, hypertension, atrial fibrillation, and ischemic heart disease), types of imaging done (CT scan, MRI, etc.), and occurrence of in-hospital pneumonia. Risk factors associated with likelihood of negative outcomes were identified.

Results: The data was highly reliable at predicting discharge to rehabilitation and all other outcomes vs. death for $\mathrm{ICH}$ (AUC 0.85 and 0.825 , respectively), all discharge outcomes except home vs. rehabilitation for ischemic stroke, and discharge home vs. others and home vs. rehabilitation for TIA (AUC 0.948 and 0.873 , respectively). Electronic health record data appeared to provide improved prediction of outcomes over stroke specific clinical factors from the machine learning models. Common risk factors associated with a negative impact on expected outcomes appeared clinically intuitive, and included older age groups, 
prior ventilatory support, urinary incontinence, need for imaging, and need for allied health input.

Conclusion: Electronic administrative records from this cohort produced reliable outcome prediction and identified clinically appropriate factors negatively impacting most outcome variables following hospital admission with stroke. This presents a means of future identification of modifiable factors associated with patient discharge destination. This may potentially aid in patient selection for certain interventions and aid in better patient and clinician education regarding expected discharge outcomes.

Keywords: electronic records, stroke outcomes, machine learning, discharge destinations, stroke mortality

\section{INTRODUCTION}

The use of electronic administrative records has become widespread in many settings in recent years. This includes the primary care setting and hospital environment (1). Administrative data in the Australian setting may be in the form of mandatory hospital collected data relating to every hospital episode of care, with the data reported to state health departments, in order to inform health care delivery, resourcing, and financial allocation (2). Administrative datasets include primary and secondary diagnosis codes, coding related to comorbidities, discharge destination, and other demographic data. The ability to harness this data to improve patient care, predict outcomes, and identify risk factors for recurrent disease and readmission means that this has become an important area for research and health metrics (3). The heterogeneity of the data and data systems themselves mean that close collaboration between clinicians and analysts is required. Identifying the type of data available and applying this to appropriate clinical questions not yet answered makes this exciting future area of endeavor. This also increases the importance of accurate data collection. Even more vital is the capture of disease specific factors.

Despite the apparent decrease in stroke incidence, in an aging population, stroke survival, and prevalence is increasing $(4,5)$. This dramatically increases the societal burden of care. Importantly, stroke outcomes are significantly affected by timely hyperacute therapies such as thrombolysis and endovascular clot retrieval for ischemic stroke (6-8), admission to a specialized stroke unit setting (9), appropriate imaging and secondary prevention therapies (10), dysphagia screening, and early mobilization (11). These interventions directly impact the need for rehabilitation or other discharge outcomes, including the potential need for long-term high-level care, and mortality (12). Understanding the factors contributing to functional outcome after stroke provides a potential target for clinicians to alter their management of patients (13). It is important to clarify if these strategies are routinely implemented through available data and audit processes, which may be best performed by disease specific quality clinical registries (14). Whilst the interventions above are well-proven to influence outcomes and also result in a reduction in hospital length of stay and readmission (15), there may be other novel factors during the admission process that have not been previously captured or studied. Analysis of available administrative data may identify process, structural, and outcome measures not previously recognized.

It is important to acknowledge the limitations of administrative datasets. Functional outcome data for stroke from administrative data may not be well-documented at any stage in the collection process. Stroke severity such as the NIHSS score may not be routinely captured or mandated and is known to directly impact outcomes $(12,15)$. Standard functional scoring such as the modified Rankin score or Barthel index may not be well-recorded and are not mandated in the electronic data. At best, in some cases, we may only be able to use proxy markers of function, such as in-hospital mortality, or discharge destination. Whilst these surrogate outcomes are well-captured from administrative data, they may not illustrate functional status comprehensively and in particular relation to stroke outcomes, do not inform around the 3- or 12-month clinical status, often used to assess the benefits of interventions in stroke patients. However, the systematic methods used, relatively complete capture of admitted patient data and system wide data collection in administrative datasets make these compelling sources to utilize.

Using machine learning techniques to answer health related questions presents a unique and powerful option for improving diagnosis, treatment, and outcome measures. There are also opportunities for identifying predictive factors impacting patient outcomes. Knowledge regarding patient and other factors associated with certain outcomes may allow future application of measures that influence patient care.

\section{AIMS}

We sought to use data from existing electronically collected administrative records to identify risk factors associated with specific outcomes for patients with stroke (both ischemic and hemorrhagic) admitted to a large regional hospital, in Victoria, Australia. In addition, we sought to evaluate the utility of using a large array of available electronic health record data from a cohort of patients, when compared to a cohort of patients with available stroke specific clinical factors, to predict discharge outcomes following hospital admission with stroke, using machine learning techniques. 


\section{METHODS}

\section{Study Setting}

Barwon Health is a large regional tertiary hospital, located in Geelong, approximately $1 \mathrm{~h}$ to the west of Melbourne, the second most populous city in Australia. This health service provides public hospital care to the population of Geelong and surrounding regional areas. The hospital includes a comprehensive neurology service, including acute stroke thrombolysis, dedicated specialized and geographically located stroke unit, and high-level imaging facilities available for acute stroke investigation. The benefits of evaluating this patient cohort include that the majority of patients with stroke are admitted to the public hospital, via the emergency department, rather than local private hospitals. Nearly all cases were likely to be captured for this region as a result. Stroke units in Australia do not currently require formal stroke unit certification, however, designated stroke units are required to adhere to a number of key elements defined in the national stroke services framework (16).

We obtained a comprehensive selection of data fields from the routinely collected electronic administrative data from Barwon Health, for the period 2003-2014. Administrative data refers to both coding and demographic data and is reportable to the state Department of Health and Human Services $(2,17)$. We analyzed data based on all patients with an admission diagnosis of stroke, using ICD 10 coding nomenclature. Due to the lack of stroke specific data on functional outcomes after the incident event, surrogate outcomes of discharge destination, and inhospital mortality were thought to be the most appropriate markers of outcome. Comparisons were made between patient admission source i.e., from home, rehabilitation, nursing home, other hospital, and discharge destination, including death in hospital. The comparisons were performed in order of perceived severity of the outcome. Patient admission source is a defined variable collected for all hospital admitted episodes, as opposed to their discharge destination. By ascertaining relevant factors contributing positively or negatively to our defined outcomes, we hoped to be able to understand novel patient, investigation, and management factors associated with our outcomes. Prior ethics approval had been provided for all data use and analysis between Barwon Health and Deakin University in an institutional agreement.

\section{Dataset}

The patient cohort consisted of 2,531 patients with confirmed diagnosis of Stroke or TIA admitted between July 2009 and June 2013. A stroke admission was defined by ICD-10 codes G46, I6069, G450-453, and G458-459 in the discharge diagnoses (either primary or secondary). For each patient, the index admission was defined as the first stroke admission of the patient starting from 1st January 2009. Patient records available from Barwon Health admissions prior to the index admission were available and were used to construct independent variables. Available data from index admissions and prior admissions included all data reportable to the state Department of Health and Human Services as part of mandatory hospital reporting $(2,17)$. Our dataset was not able to capture admission data outside of Barwon Health admissions i.e., was not linked to private hospital admissions or admissions to other public institutions. The outcome considered was the discharge destination (home, rehabilitation, or nursing home) if the patient is alive, or death if the patient had died during hospitalization.

\section{Data Analysis}

We considered all available administrative hospital data including static information (age, gender, occupation, insurance types), and time-stamped events associated with emergency visits, hospitalizations, radiological tests, length-of-stay, emergency attendance time, primary and secondary diagnoses, and procedures. The use of cerebral imaging such as CT and MRI in stroke evaluation is an important process measure in helping to accurately diagnose and manage patients and was felt important to include in the analysis. Medication usage data was not available from our dataset. Age was coded as a binary variable (i.e., the age variable or not) in one of 10 -year intervals, in line with other stroke community and cohort studies $(18,19)$. Occupation was a binary of value 1 if it was either pensioner, retired, or home duties and 0 otherwise. Time-stamped events were aggregated over two periods of time prior to the index admission: $0-12$ months and beyond 12 months. This resulted in a total of 1,303 features. Models were built to analyse the factors associated with different outcomes [e.g., in-hospital death vs. others (i.e., Discharge to home, Rehabilitation, Nursing home), Discharge to home vs. others] using penalized logistic regression with Lasso (20).

We split the data in time (external validation) with data from July 2009 to June 2012 for derivation of predictive models and July 2012 to June 2013 as validation. Confidence intervals were computed based on 100 bootstrapped derivation cohorts from the original derivation cohorts using sampling with replacement.

Five different comparison settings for each of the three subcohorts of stroke [intracerebral hemorrhage (ICH), ischemic stroke, transient ischemic attack (TIA)] are considered, by evaluating factors likely to be associated with the defined outcomes, vs. other outcomes.

- Discharge to home vs. others (rehabilitation, nursing home, in-hospital death) out of all patients

- Discharge to rehabilitation vs. home for patients either discharged to home or rehabilitation

- Discharge to nursing home vs. rehabilitation for patients either discharged at nursing home or rehabilitation

- Discharge to nursing home vs. death

- In hospital death vs. discharge to all other places (home, rehabilitation, nursing home)

Where there were small sample sizes, data were collapsed together for the purposes of comparison.

All data processing was performed off-line using a commercial software package (MATLAB, Statistics Toolbox, The MathWorks Inc., 1994-2014). Prediction accuracy is expressed as the area under the receiver operating characteristic curve (AUC). Missing data were imputed.

Two feature sets were constructed: 
1. Features constructed from the electronic administrative record which included all available detailed diagnosis, procedure, and administrative data. This included stroke and TIA related diagnostic codes (I60-I69, G45) relating to primary diagnosis, secondary (comorbidity) diagnostic codes, and all available procedure codes relating to patient admissions. The number of variables was 1,303 (some examples of the types of features included can be seen in the data items listed in the Appendix Figures).

2. Features constructed from more stroke specific clinical data such as age, gender, smoking habits, co-morbidities (hyperlipidaemia, diabetes, hypertension, atrial fibrillation, and ischemic heart disease), types of imaging done (CT scan, MRI, etc.,-an important stroke management process marker), and occurrence of in-hospital pneumonia. Specific stroke risk factors such as alcohol use, anticoagulant use, and obesity are not included in the routine data collection.

\section{RESULTS}

We derived prediction results for three subcohorts of stroke patients (ICH, ischemic stroke, and TIA) in five different settings, as outlined above. All results presented are based on the validation cohort, unless otherwise specified. Patient characteristics and discharge destinations are summarized in Tables 1, 2.

The percentage of stroke type found in our cohort is similar to other cohorts. The occurrence of "Not specified" diagnostic codes highlights a key problem in using administrative datasets and is identified as a limitation in other cohort studies (21).

The percentage of patients with specified comorbidities is again similar to other cohort studies $(4,22)$, although the percentage with IHD was lower. In relation to imaging, $100 \%$ of patients underwent imaging with CT scan of the brain, as is standard clinic practice in patients with suspected stroke or TIA, in order to ascertain presence of infarction or hemorrhage, as well as other causes of potential stroke mimics. The majority of patients had a length of stay of between 1 and 5 days, in keeping with findings from local acute stroke audits.

We sought to identify specific predictive factors from our analysis associated with the outcomes we have studied. These factors were items from our administrative data, presented in the figures below as both positively and negatively weighted variables. Table 6 below summarizes factors found to negatively impact the outcome presented. For example, for patients with $\mathrm{ICH}$, patients were less likely to be discharged home vs. to all other discharge destinations (rehabilitation, nursing home, or die in hospital) in older age groups (80-90 years old), had had prior ventilatory support, a history of urinary incontinence, or diagnosis of SAH.

Figures in the Appendix below identify all factors from the administrative dataset that both positively and negatively impact the outcomes being studied and represent weights of the linear model.
TABLE 1 | Patient characteristics.

\begin{tabular}{|c|c|c|}
\hline No. of patients & 2,531 & $\%$ \\
\hline Males & 1,346 & 53.2 \\
\hline Females & 1,185 & 46.8 \\
\hline Mean age & 72.9 (18.4-99.8) & \\
\hline$<50$ years & & 1.5 \\
\hline $50-59$ years & & 9.6 \\
\hline 60-69 years & & 24.5 \\
\hline 70-79 years & & 24.2 \\
\hline 80-89 years & & 32.2 \\
\hline 90-99 years & & 8.0 \\
\hline \multicolumn{3}{|l|}{ Stroke type } \\
\hline Transient ischemic attack & & 25.1 \\
\hline Intracerebral hemorrhage & & 14.6 \\
\hline Ischemic stroke & & 37.7 \\
\hline Aneurysm & & 0.9 \\
\hline Not specified & & 21.8 \\
\hline \multicolumn{3}{|l|}{ Comorbidities } \\
\hline Hypertension & & 52.5 \\
\hline Atrial fibrillation & & 15.4 \\
\hline Hyperlipidaemia & & 6.7 \\
\hline Smoking & & 13.8 \\
\hline Ischemic heart disease & & 8.4 \\
\hline \multicolumn{3}{|l|}{ Imaging } \\
\hline CT brain & & 100 \\
\hline$X$ ray chest & & 91.8 \\
\hline US carotid doppler & & 43.8 \\
\hline MRI brain & & 36.8 \\
\hline \multirow[t]{2}{*}{ Length of stay } & $1-5$ days & 99.1 \\
\hline & $>5$ days & 0.9 \\
\hline
\end{tabular}

TABLE 2 | Discharge destination.

\begin{tabular}{lc}
\hline Discharge destinations & $\%$ \\
\hline Home & 58.9 \\
Rehabilitation & 24.5 \\
Nursing home & 5.1 \\
In hospital death & 9.4
\end{tabular}

\section{DISCUSSION}

Our goal was to compare the utilization of an electronic health record model constructed using a general set of coding data and demographic data, with a model based on a specifically selected set of clinically recognized features, in identifying data reliability, predict discharge destination, and identify risk factors associated with specific outcomes following hospital admission with stroke. Analysis using the electronic health record data provided better prediction of outcome and use of stroke specific factors did not appear to improve the model's reliability. When comparing the data from Tables 4, 5, our data was highly reliable in predicting outcomes in patients with $\mathrm{ICH}$ of discharge to rehabilitation vs. nursing home or death, as well as all other discharge 
TABLE 3 | Percentage of patients that fit the model in the derivation cohort under five different prediction settings for three sub-cohorts of stroke.

\begin{tabular}{llll}
\hline & $\begin{array}{l}\text { Intracerebral } \\
\text { hemorrhage }\end{array}$ & Ischemic stroke & TIA \\
\hline Home vs. Others & $16.5 \%(357)$ & $47.1 \%(830)$ & $87.5 \%(659)$ \\
Home vs. rehab & $24.6 \%(240)$ & $57.8 \%(677)$ & $93.8 \%(531)$ \\
$\begin{array}{l}\text { Rehab vs. nursing } \\
\text { home or death (nursing } \\
\text { home and death }\end{array}$ & $60.7 \%(298)$ & $65.2 \%(437)$ & \\
$\begin{array}{l}\text { collapsed due to small } \\
\text { sample size) }\end{array}$ & & & \\
$\begin{array}{l}\text { Nursing home vs. } \\
\text { death }\end{array}$ & $17.8 \%(117)$ & $35.3 \%(153)$ & \\
$\begin{array}{l}\text { Others vs. death } \\
26.9 \%(357)\end{array}$ & $11.9 \%(830)$ & \\
\hline
\end{tabular}

Where there are missing outcomes in the table, this denotes scenarios where derivation is difficult due to the lack of sufficient number of patients. The numbers in parentheses denote total patient numbers in the derivation category for that pair of outcomes.

outcomes vs. death. In ischemic stroke, the data was reliable at predicting discharge home vs. other outcomes, discharge to rehabilitation vs. nursing home or death, discharge to nursing home vs. death, and all other outcomes vs. death. For TIA, the data proved reliable in predicting discharge home and to home vs. rehabilitation.

There are several problems in using electronic administrative records data to identify risk factors and predict outcomes. The amount of electronic data collection contained in these datasets is copious, and there is significant risk in misinterpreting data if it is not disease specific. The complexities of interactions between patient demographic, diagnostic, imaging, procedural, and outcome data may be difficult to interpret. If there is a small group of well-known risk factors, which have been expertly evaluated or have a sound scientific or peer reviewed connection with the research question or patient group, this may be applied in the analysis. Another method may be to examine a larger group of risk factors and determine their statistical significance and predictive power, and hence refine these to the patient population, using regression methods. However, this method again may not be disease specific. The risk factors used in any analysis may be too limited for the data available, and too much data may make the results noisy or uninterpretable. There are inherent differences in risk factors, measures of severity, and specific management strategies for ischemic stroke/TIA and hemorrhagic stroke, which may be useful to capture in any comprehensive medical record.

The use of logistic regression with Lasso is a common linear classifier method that is also suitable for feature selection. The models obtained are likely to be more parsimonious than logistic regression alone. Our aim was to contribute to understanding about the utility of using electronic health record data for clinical prediction, rather than use of different machine learning methods.

Although we understand risk factors such as age, gender, and co-morbidities well in terms of their likely effect on outcomes in stroke patients, the highly detailed data collected by the hospital data warehouse, both for reporting, planning, and financial purposes, means there are likely to be novel but useful predictive factors identified from analyses like this one. Of interest from our list of identified predictive factors for discharge destination were the findings of prior factors in patient histories including prior ventilatory support, imaging factors, respiratory and urinary tract conditions, and allied health input. These novel past history and other elements may indicate new and innovative areas to focus on, guiding clinically, and patient relevant insights and exploration.

Note that factors for Nursing Home vs. Rehabilitation and Death vs. Others for patients with TIA are not presented since the predictive models are unstable (as seen by the lack of valid data in Table 3).

The burden of stroke is significant, and recurrent events may add significantly to pre-existing disability, with further acute healthcare, career, and economic impact. Being able to better identify factors associated with poorer outcome can help clinicians intensify efforts in certain areas. Predictive measures can be factored into clinical care paradigms in situations where the data is reliable and serve as an additional tool.

Many of the identified factors from the model felt to influence the outcomes in question appear clinically intuitive. Older age group, the need for allied health and complications of illness such as pneumonitis the clinician understands have a substantial impact on good outcomes in patients with stroke and other diseases. However, understanding these specific factors may help us to better define which patients require more attention or intervention, and supports the strength of the dataset. Some of these factors are not modifiable but can help us in prognostication and better informing patients and families.

One of the limitations of this study was the lack of an available functional outcome measure in the electronic data, leading to the use of "surrogate" markers of function on discharge from the acute event. The use of clinically important scores such as the modified Rankin score and NIHSS (23) in most stroke outcome studies is not possible using the current dataset and highlights the important areas of deficit in clinically relevant/disease specific measures from administrative data. The lack of important imaging data such as stroke infarct volume, and stroke specific treatments, is also a barrier.

\section{CONCLUSION}

The electronic administrative record data for our stroke cohort appeared reliable in outcome prediction for most patients and for different stroke types, when based on discharge destination. Risk factors having a negative impact on the defined discharge destinations provide useful and intuitive patient factors which could allow therapeutic intervention and a clearer understanding of which patients are more likely to have better clinical outcomes following an index stroke. In 
TABLE 4 | AUC of prediction for three different sub-cohorts of stroke at five different settings.

\begin{tabular}{|c|c|c|c|}
\hline & Intracerebral hemorrhage & Ischemic stroke & TIA \\
\hline Home vs. others & $0.604(0.404-0.791)$ & $0.803(0.746-0.891)$ & $0.948(0.901-0.955)$ \\
\hline Home vs. rehab & $0.600(0.418-0.783)$ & $0.752(0.683-0.820)$ & $0.873(0.749-0.996)$ \\
\hline Rehab vs. nursing home or death & $0.850(0.737-0.963)$ & $0.818(0.736-0.801)$ & \\
\hline Nursing home vs. death & $0.550(0.245-0.855)$ & $0.902(0.777-1.00)$ & \\
\hline Others vs. death & 0.825 (0.698-0.952) & 0.881 (0.804-0.959) & \\
\hline
\end{tabular}

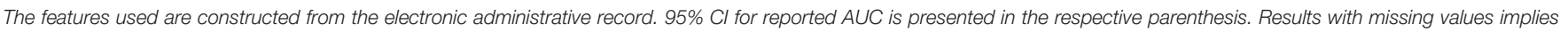
invalid $\mathrm{Cl}$ associated with unstable models, generally resulted from lack of sufficient data.

TABLE 5 | AUC of prediction for three different sub-cohorts of stroke at five different settings.

\begin{tabular}{|c|c|c|c|}
\hline & Intracerebral hemorrhage & Ischemic stroke & TIA \\
\hline Home vs. others & $0.459(0.285-0.634)$ & $0.702(0.634-0.769)$ & $0.794(0.585-1.00)$ \\
\hline Home vs. rehab & $0.296(0.131-0.462)$ & $0.636(0.558-0.714)$ & $0.729(0.283-0.996)$ \\
\hline $\begin{array}{l}\text { Rehab vs. nursing } \\
\text { home or death }\end{array}$ & $0.504(0.346-0.661)$ & $0.767(0.674-0.860)$ & \\
\hline $\begin{array}{l}\text { Nursing home vs. } \\
\text { death }\end{array}$ & $0.625(0.369-0.881)$ & $0.778(0.586-0.970)$ & \\
\hline Others vs. death & $0.583(0.424-0.742)$ & 0.808 (0.718-0.899) & \\
\hline
\end{tabular}

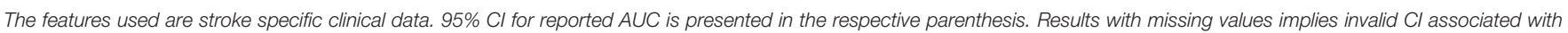
unstable models, generally resulted from lack of sufficient data.

TABLE 6 | Selected predictive factors associated with the prediction models.

\begin{tabular}{|c|c|c|c|c|c|}
\hline & $\begin{array}{l}\text { Discharge home vs. } \\
\text { other outcomes }\end{array}$ & $\begin{array}{l}\text { Discharge home vs. } \\
\text { to rehabilitation }\end{array}$ & $\begin{array}{l}\text { Discharge to } \\
\text { rehabilitation vs. } \\
\text { nursing home or } \\
\text { death }\end{array}$ & $\begin{array}{l}\text { Discharge to nursing } \\
\text { home vs. death }\end{array}$ & $\begin{array}{l}\text { All other discharge } \\
\text { outcomes vs. death }\end{array}$ \\
\hline $\mathrm{ICH}$ & $\begin{array}{l}\text { Older age group } \\
(80-90), \text { prior } \\
\text { ventilatory support, } \\
\text { urinary incontinence, } \\
\text { SAH }\end{array}$ & $\begin{array}{l}\text { SAH, prior ventilatory } \\
\text { support, prior CT } \\
\text { imaging, urinary } \\
\text { incontinence, older age } \\
\text { group }\end{array}$ & $\begin{array}{l}\text { Prior admission from } \\
\text { emergency to the ward, } \\
\text { prior CT brain/cervical } \\
\text { spine, older age group, } \\
\text { prior ventilatory support }\end{array}$ & $\begin{array}{l}\text { Prior ventilatory } \\
\text { support, age } 70-80 \text {, } \\
\text { male gender, SAH }\end{array}$ & $\begin{array}{l}\text { Ventilatory support, age } \\
>90 \text {, prior CT } \\
\text { brain/cervical spine, } \\
\text { age } 80-90, \text { past } \\
\text { admission from } \\
\text { emergency to ward }\end{array}$ \\
\hline Ischemic stroke & $\begin{array}{l}\text { Urinary retention, } \\
\text { hemiplegia, age group } \\
\text { 80-90, allied health } \\
\text { input as inpatient, chest } \\
\text { X-ray, pneumonitis }\end{array}$ & $\begin{array}{l}\text { Urinary retention, } \\
\text { inpatient allied health } \\
\text { involvement, } \\
\text { hemiplegia, older age } \\
\text { group (80-90) }\end{array}$ & $\begin{array}{l}\text { Older age group (>90), } \\
\text { pneumonitis, other } \\
\text { intestinal disorders, and } \\
\text { restlessness/agitation }\end{array}$ & $\begin{array}{l}\text { Other medical care } \\
(\mathrm{Z} 51)^{\star}, \text { prior ventilatory } \\
\text { support, pneumonitis, } \\
\text { unspecified threat to } \\
\text { breathing, chest X-ray, } \\
\text { and hemiplegia }\end{array}$ & $\begin{array}{l}\text { Other medical care, } \\
\text { pneumonitis, chest } \\
\text { X-ray, and unspecified } \\
\text { threat to breathing }\end{array}$ \\
\hline $\mathrm{TIA}$ & $\begin{array}{l}\text { Older age group (>90), } \\
\text { cerebral infarction } \\
\text { diagnosis, } \\
\text { disorientation, prior } \\
\text { allied health care and } \\
\text { diagnosis of } \\
\text { syncope/collapse }\end{array}$ & $\begin{array}{l}\text { Older age group (>90), } \\
\text { diagnosis of cerebral } \\
\text { infarction, } \\
\text { syncope/collapse, and } \\
\text { prior allied health } \\
\text { involvement }\end{array}$ & $N / A^{*}$ & $N / A^{*}$ & $N / A^{*}$ \\
\hline
\end{tabular}

Factors are those having a negative impact on the outcome in question.

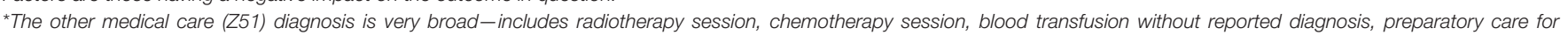
subsequent treatment, palliative care, desensitization to allergens, other specified medical care, medical care unspecified. 
future, the availability of more stroke specific clinical factors in the dataset, including better clinical outcome variables, will likely aid in improving the validity of our data for analysis and prediction.

\section{DATA AVAILABILITY STATEMENT}

The datasets presented in this article are not readily available because the raw data outputs are no longer available due to changes in University and health service

\section{REFERENCES}

1. Dregan A, Toschke MA, Wolfe CD, Rudd A, Ashworth M, Gulliford MC. Utility of electronic patient records in primary care for stroke secondary prevention trials. BMC Public Health. (2011) 11:86. doi: 10.1186/1471-2458-11-86

2. Victorian Admitted Episodes Dataset: Department of Health and Human Services, State Government of Victoria. (2015). Available online at: https:// www2.health.vic.gov.au/hospitals-and-health-services/data-reporting/ health-data-standards-systems/data-collections/vaed (accessed June, 2015).

3. Frisher M, Short D, Bashford J. Determining patient characteristics for decision analysis support systems using anonymized electronic patient records. Health Informatics J. (2010) 16:4957. doi: 10.1177/1460458209353559

4. Clissold BB, Sundararajan V, Cameron P, McNeil J. Stroke incidence in Victoria, Australia-emerging improvements. Front Neurol. (2017) 8:180. doi: 10.3389/fneur.2017.00180

5. Jamrozik K, Broadhurst RJ, Lai N, Hankey GJ, Burvill PW, Anderson CS. Trends in the incidence, severity, and short-term outcome of stroke in perth, Western Australia. Stroke. (1999) 30:2105-11. doi: 10.1161/01.STR.30.10.2105

6. The National Institute of Neurological Disorders and Stroke rt-PA Stroke Study Group. Tissue plasminogen activator for acute ischemic stroke. N Engl J Med. (1995). 333:1581-7. doi: 10.1056/NEJM199512143332401

7. Goyal M, Menon BK, van Zwam WH, Dippel DW, Mitchell PJ, Demchuk AM, et al. Endovascular thrombectomy after large-vessel ischaemic stroke: a metaanalysis of individual patient data from five randomised trials. Lancet. (2016) 387:1723-31. doi: 10.1016/S0140-6736(16)00163-X

8. Hacke W, Kaste M, Bluhmki E, Brozman M, Davalos A, Guidetti D. et al. Thrombolysis with alteplase 3 to 45 hours after acute ischemic stroke. $N$ Engl J Med. (2008) 359:1317-29. doi: 10.1056/NEJMoa0804656

9. Stroke Unit Trialists' Collaboration. Organised inpatient (stroke unit) care for stroke. Cochrane Database Syst Rev. (2013) 2013:CD000197. doi: 10.1002/14651858.CD000197.pub3

10. Hankey GJ. Preventable stroke and stroke prevention. J Thromb Haemost. (2005) 3:1638-45. doi: 10.1111/j.1538-7836.2005.01427.x

11. Fjaertoft H, Indredavik B, Magnussen J, Johnsen R. Early supported discharge for stroke patients improves clinical outcome. Does it also reduce use of health services and costs? One-year follow-up of a randomized controlled trial. Cerebrovasc Dis. (2005) 19:376-83. doi: 10.1159/000085543

12. Elwood D, Rashbaum I, Bonder J, Pantel A, Berliner J, Yoon S, et al. Length of stay in rehabilitation is associated with admission neurologic deficit and discharge destination. PM R. (2009) 1:147-51. doi: 10.1016/j.pmrj.2008.10.010

13. Frank M, Conzelmann M, Engelter S. Prediction of discharge destination after neurological rehabilitation in stroke patients. Eur Neurol. (2010) 63:22733. doi: 10.1159/000279491

14. Registry ASC. AUSCR (2016). Available online at: www.auscr.com.au (accessed June, 2013).

15. Ruuskanen EI, Laihosalo M, Kettunen J, Losoi H, Nurmi L, Koivisto $\mathrm{AM}$, et al. Predictors of discharge tohome after thrombolytic treatment agreements. Requests to access the datasets should be directed tobenc73@hotmail.com.

\section{AUTHOR CONTRIBUTIONS}

All authors contributed to conception and design of the study. SR, WL, TT, DP, and BC organized the database. SR, WL, TT, $\mathrm{DP}$, and SV performed the statistical analysis. BC wrote the first draft of the manuscript. All authors contributed to manuscript revision, read, and approved the submitted version. in right hemisphere infarct patients. J Cent Nerv Syst Dis. (2010) 2:739. doi: 10.4137/JCNSD.S6411

16. National Stroke Services Frameworks: Stroke Foundation. (2017). Available online at: https://strokefoundation.org.au/what-we-do/treatment-programs/ clinical-guidelines/national-stroke-services-frameworks (accessed June, 2017).

17. Victorian Emergency Minimum Dataset: Department of Health and Human Services, State Government of Victoria. (2015). Available online at: https:// www2.health.vic.gov.au/hospitals-and-health-services/data-reporting/ health-data-standards-systems/data-collections/vemd (accessed June, 2015).

18. Thrift AG, Dewey HM, Macdonell RA, McNeil JJ, Donnan GA. Stroke incidence on the east coast of Australia: the North East Melbourne Stroke Incidence Study (NEMESIS). Stroke. (2000) 31:2087-92. doi: 10.1161/01.STR.31.9.2087

19. Leyden JM, Kleinig TJ, Newbury J, Castle S, Cranefield J, Anderson $\mathrm{CS}$, et al. Adelaide stroke incidence study: declining stroke rates but many preventable cardioembolic strokes. Stroke. (2013) 44:1226-31. doi: 10.1161/STROKEAHA.113.675140

20. Tibshirani R. Regression shrinkage and selection via the lasso. J Roy Stat Soc B Methodol. (1996) 58:267-88. doi: 10.1111/j.2517-6161.1996.tb02080.x

21. Hall R, Mondor L, Porter J, Fang J, Kapral MK. Accuracy of administrative data for the coding of acute stroke and TIAs. Canad J Neurol Sci. (2016) 43:765-73. doi: 10.1017/cjn.2016.278

22. Islam MS, Anderson CS, Hankey GJ, Hardie K, Carter K, Broadhurst R, et al. Trends in incidence and outcome of stroke in Perth, Western Australia during 1989 to 2001: the Perth Community Stroke Study. Stroke. (2008) 39:776-82. doi: 10.1161/STROKEAHA.107.493643

23. Schlegel DJ, Tanne D, Demchuk AM, Levine SR, Kasner SE. Prediction of hospital disposition after thrombolysis for acute ischemic stroke using the National Institutes of Health Stroke Scale. Arch Neurol. (2004) 61:10614. doi: 10.1001/archneur.61.7.1061

Conflict of Interest: The authors declare that the research was conducted in the absence of any commercial or financial relationships that could be construed as a potential conflict of interest.

Publisher's Note: All claims expressed in this article are solely those of the authors and do not necessarily represent those of their affiliated organizations, or those of the publisher, the editors and the reviewers. Any product that may be evaluated in this article, or claim that may be made by its manufacturer, is not guaranteed or endorsed by the publisher.

Copyright (C) 2021 Rana, Luo, Tran, Venkatesh, Talman, Phan, Phung and Clissold. This is an open-access article distributed under the terms of the Creative Commons Attribution License (CC BY). The use, distribution or reproduction in other forums is permitted, provided the original author(s) and the copyright owner(s) are credited and that the original publication in this journal is cited, in accordance with accepted academic practice. No use, distribution or reproduction is permitted which does not comply with these terms. 


\section{APPENDIX}

Factors for Discharge to Nursing Home vs Rehabilitation and Death vs All Other Discharge Destinations for Patients With TIA Are Not Presented as the Predictive Model Is Unstable.

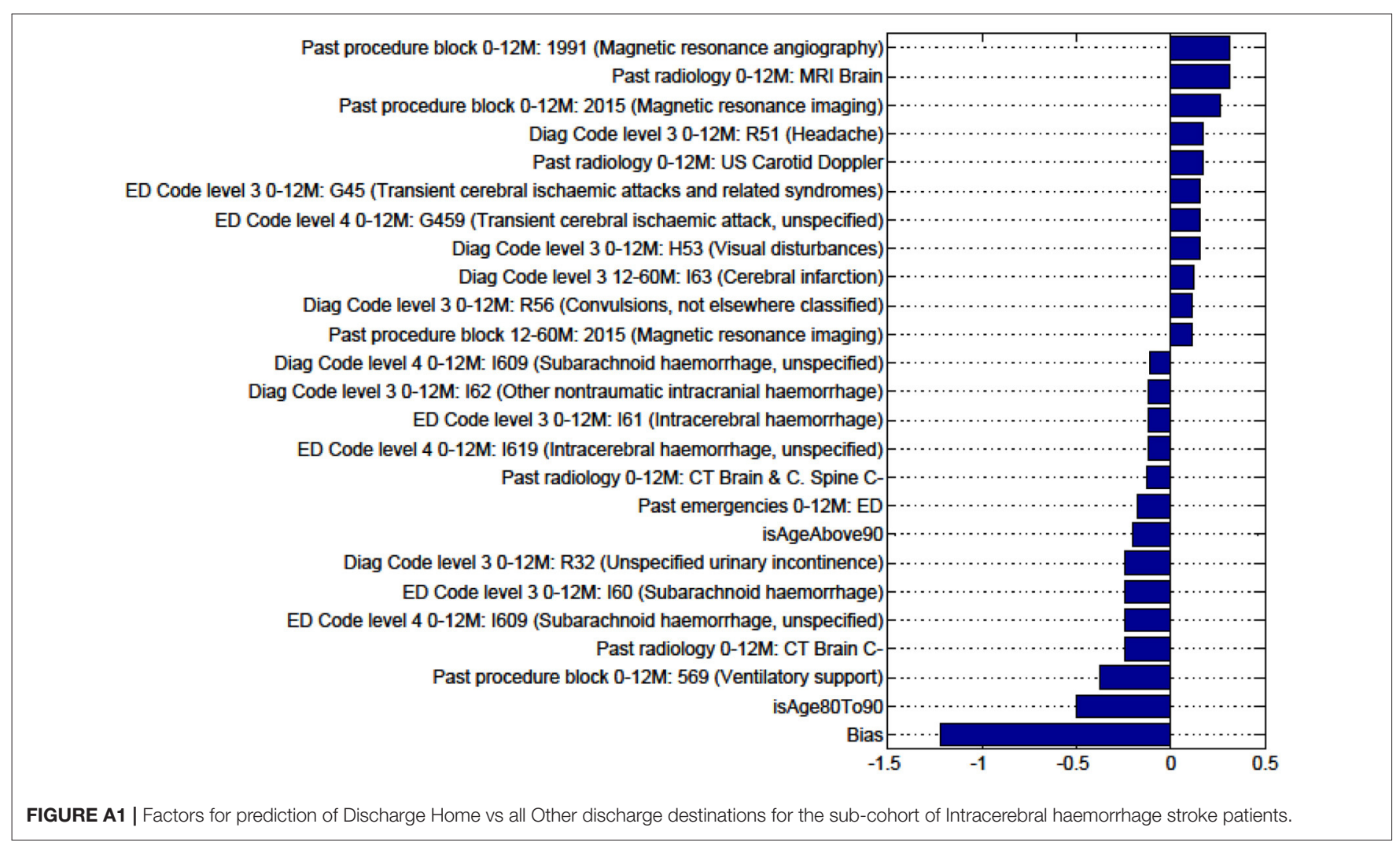




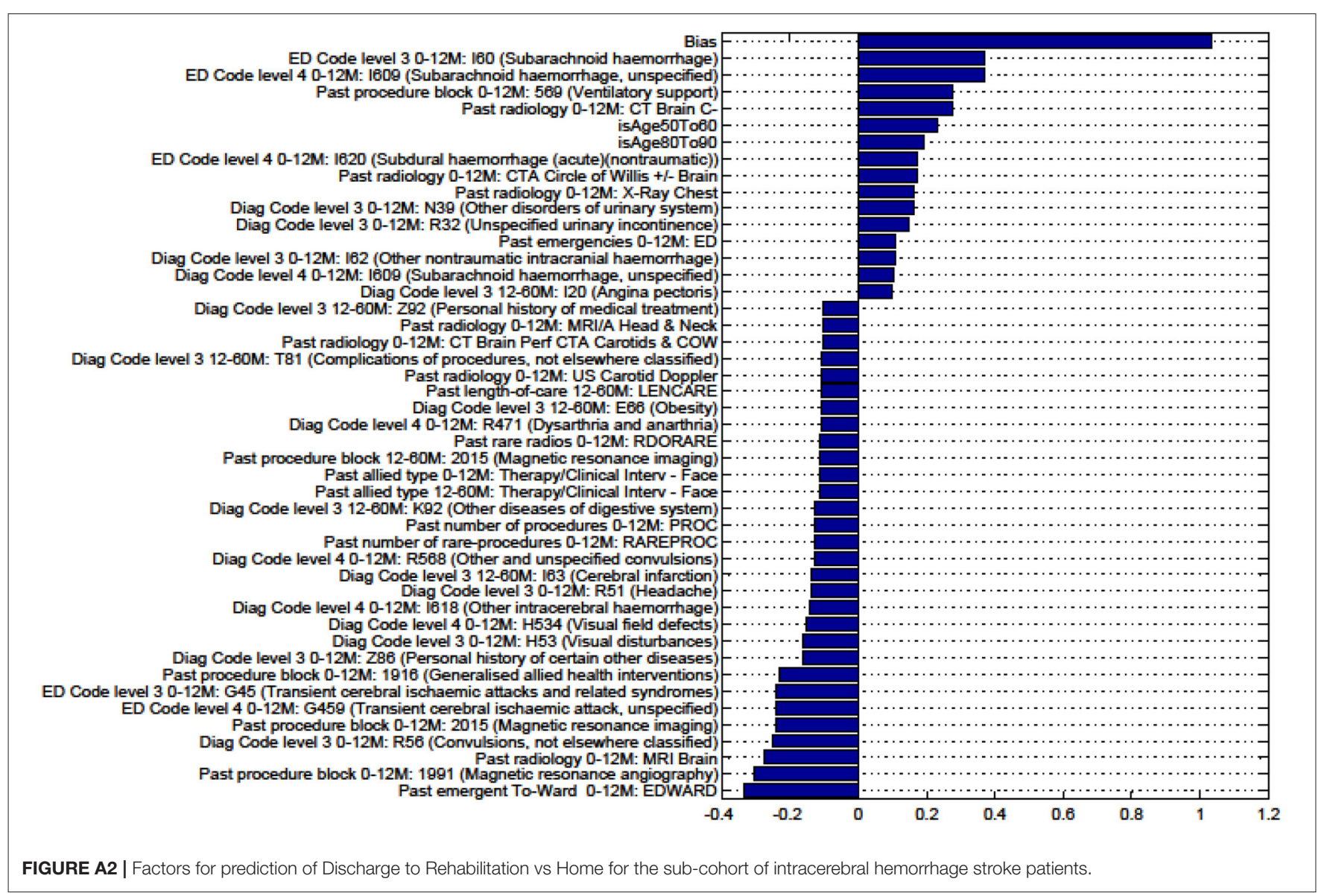




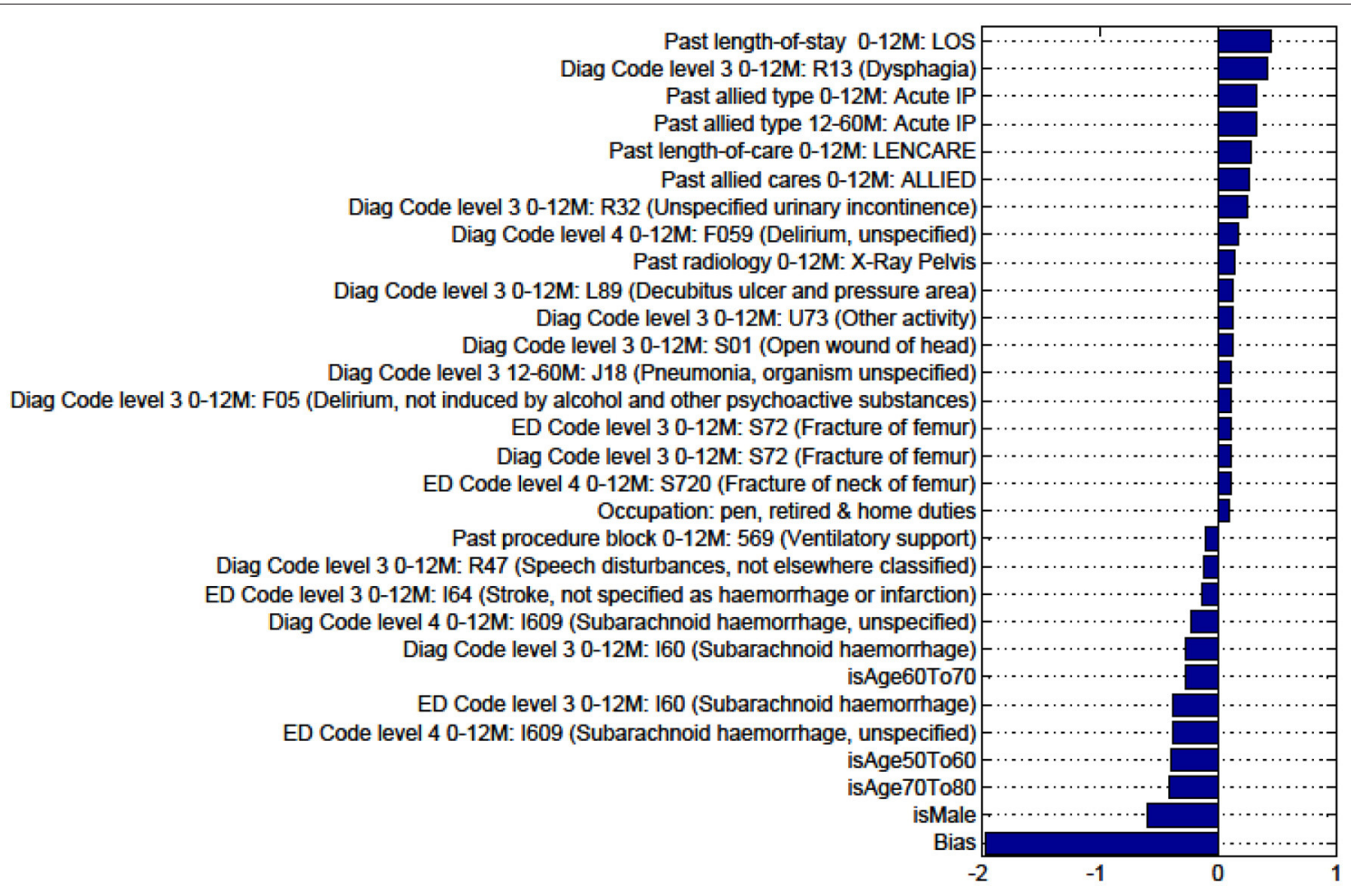

FIGURE A3 | Factors for prediction of Discharge to Nursing Home vs Rehabilitation for the sub-cohort of intracerebral hemorrhage stroke patients.

Past procedure block 0-12M: 569 (Ventilatory support) Past radiology 0-12M: CT Brain \& C. Spine C is AgeAbove 90 isAge80To90

Past emergent To-Ward 0-12M: EDWARD

ED Code level 3 0-12M: 161 (Intracerebral haemorrhage) ED Code level 4 0-12M: 1619 (Intracerebral haemorrhage, unspecified) Diag Code level 3 0-12M: R40 (Somnolence, stupor and coma)

Diag Code level 4 0-12M: R470 (Dysphasia and aphasia) Past procedure block 12-60M: 1916 (Generalised allied health interventions) ED Code level 3 0-12M: 164 (Stroke, not specified as haemorrhage or infarction) Diag Code level 4 0-12M: 1619 (Intracerebral haemorrhage, unspecified) Past radiology 0-12M: CTA Circle of Willis +/-Brain isMale Past radiology 0-12M: MRI Brain Past length-of-care 0-12M: LENCARE ED Code level 3 0-12M: 160 (Subarachnoid haemorrhage) ED Code level 4 0-12M: 1609 (Subarachnoid haemorrhage, unspecified)

Diag Code level 3 0-12M: 160 (Subarachnoid haemorrhage) Diag Code level 4 0-12M: 1609 (Subarachnoid haemorrhage, unspecified) Diag Code level 3 0-12M: H53 (Visual disturbances) Occupation: pen, retired \& home duties Diag Code level 3 0-12M: R13 (Dysphagia) isAge50To60 Bias

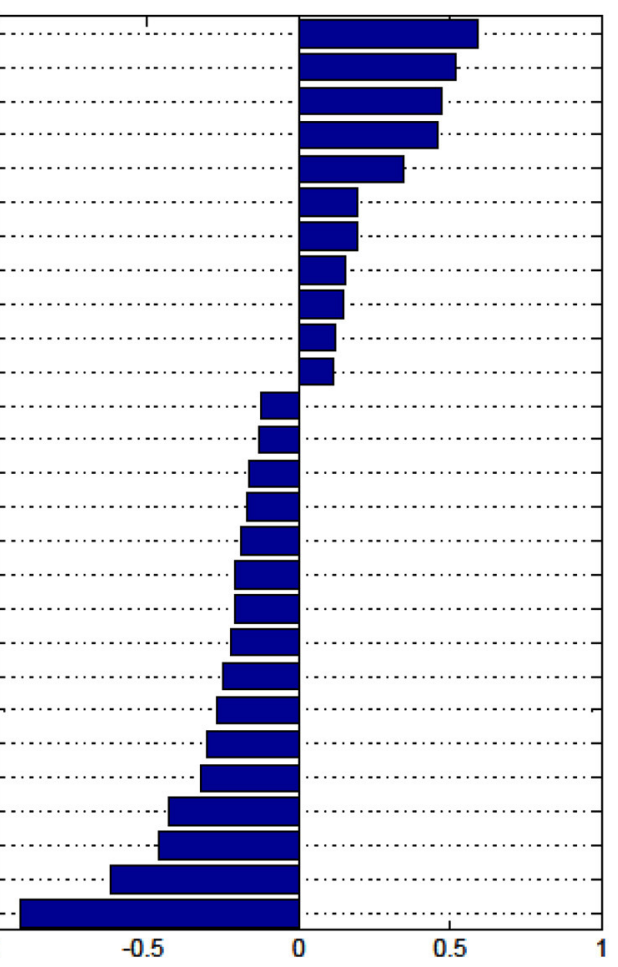

FIGURE A4 | Factors for prediction of Death vs all Other discharge destinations for the sub-cohort of intracerebral hemorrhage stroke patients. 


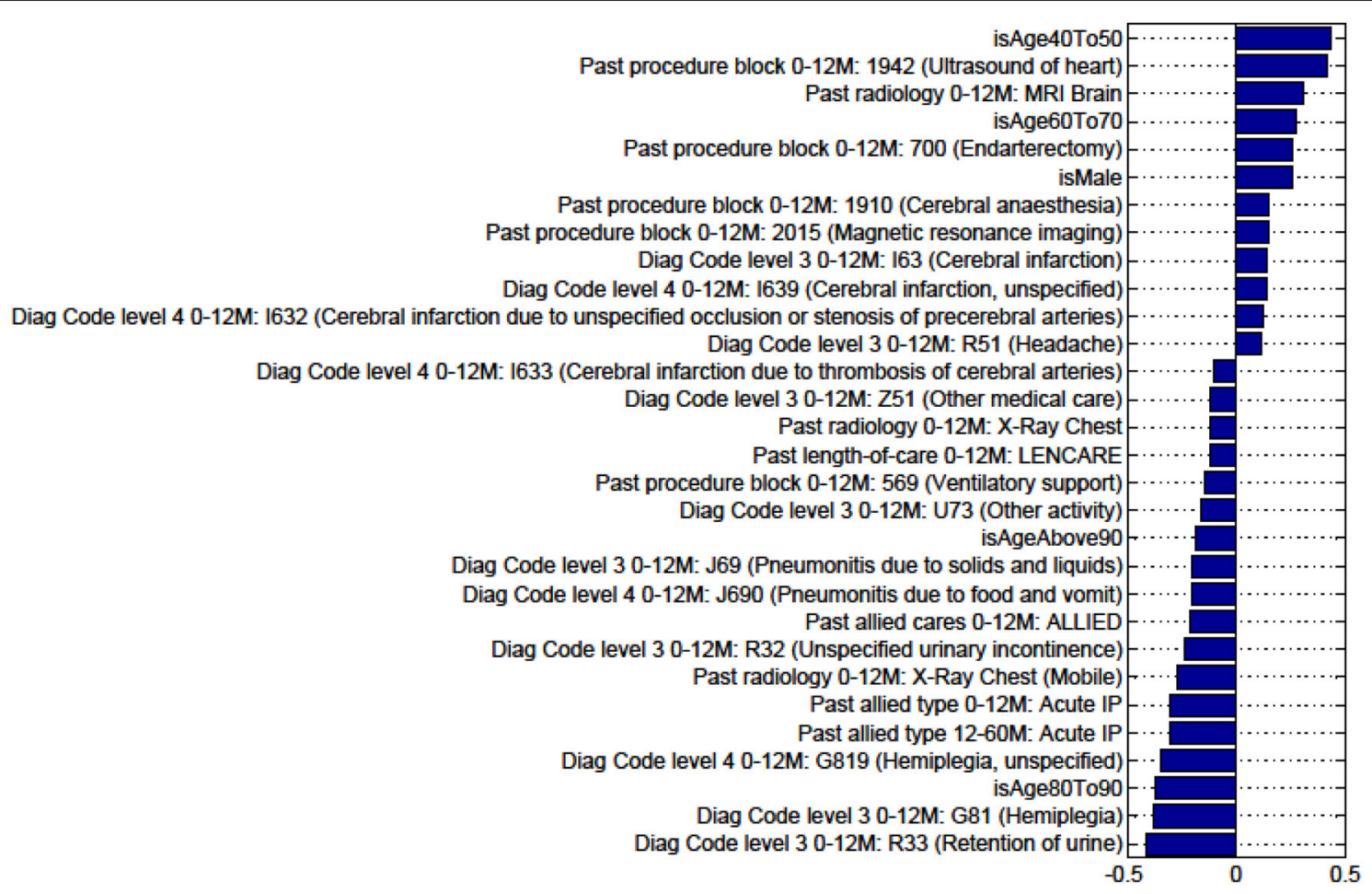

FIGURE A5 | Factors for prediction of Discharge Home vs all Other discharge destinations for the sub-cohort of ischaemic stroke patients.

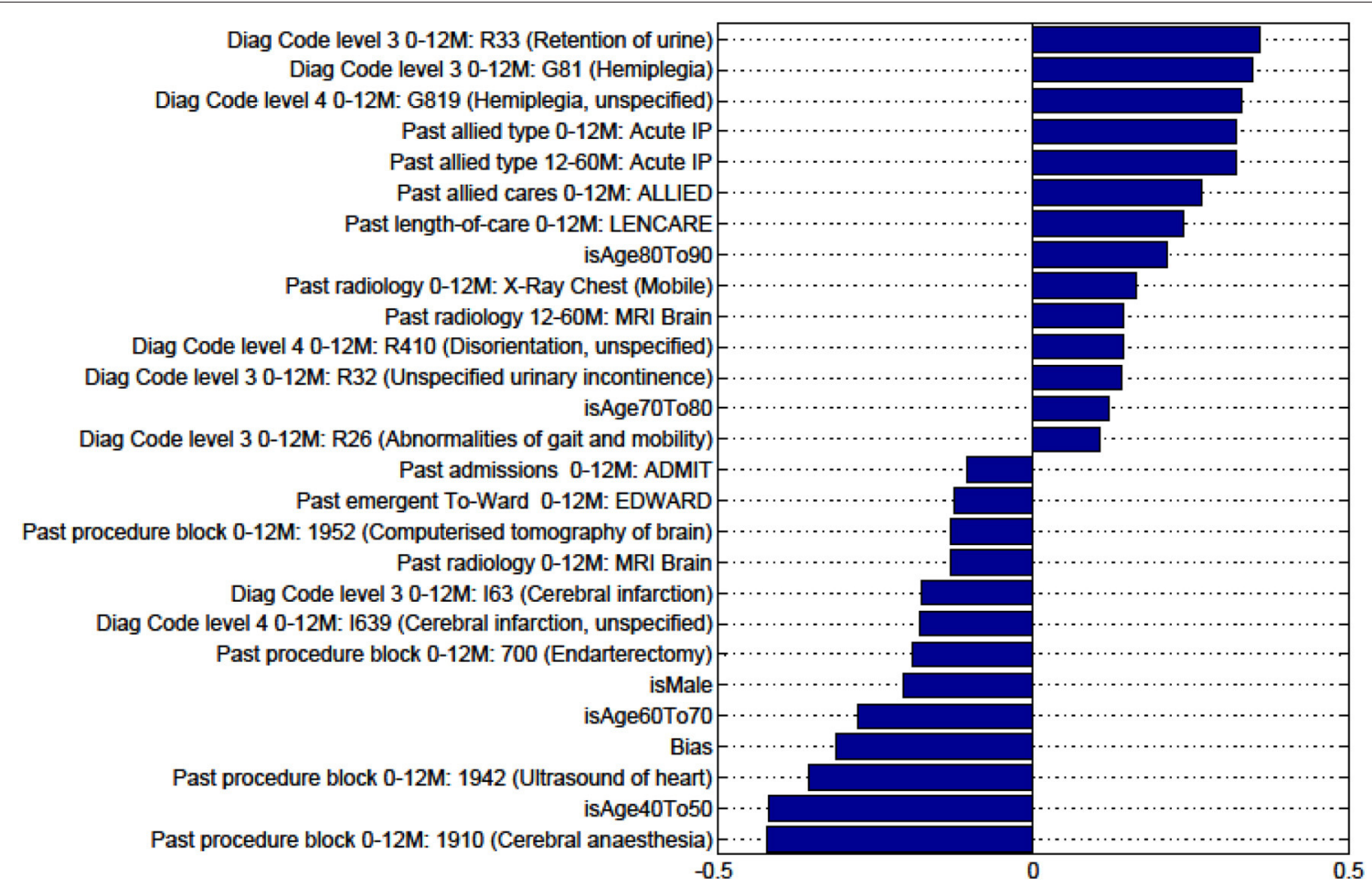

FIGURE A6 | Factors for prediction of Discharge to Rehabilitation vs Home for the sub-cohort of ischemic stroke patients. 


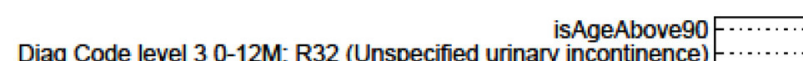

Diag Code level 3 0-12M: B96 (Other bacterial Conts as the cause of diseases classified to other chapters) Diag Code level 4 0-12M: B962 (Escherichia coli [E. coli] as the cause of diseases classified to other chapters

Diag Code level 3 0-12M: R13 (Dysphagia) Diag Code level $30-12 \mathrm{M}$ : Z75 (Problems related to medical facilities and other health care) Diag Code level 3 0-12M: N39 (Other disorders of urinary system Diag Codelevel 4 0-12M: N390 (Urinary tract infection, site not specified) Diag Code level 4 0-12M: B968 (Other specified bacterial agents as the cause of diseases classified to other chapters) Diag Code level 4 0-12M: E870 (Hyperosmolality and hypernatraemia) Diag Code level 3 0-12M: F05 (Delirium, not induced by alcohol and other psychoactive substances)

Diag Code level 4 0-12M: R451 (Restlessness and agitation Past radiology 12-60M: CT Brain CDiag Code level 3 0-12M: 86 (Volume depletion Diag Code level 4 12-60M: B962 (Escherichia coli [E. coli] as the cause of diseases classified to other chapters) Diag Code level 3 0-12M: R45 (Symptoms and signs involving emotional state) Diag Code level 3 12-60M: K59 (Other functional intestinal disorders Past procedure block 0-12M: 870 (Application, insertion or removal procedures on stomach) Diag Code level 3 0-12M: L89 (Decubitus ulcer and pressure area Past allied type 0-12M: Acute IP ED Code level 4 12-60M: N390 (Urinary Past allied type 12-60M: Acute IP Diag Code level 3 0-12M: G81 (Hemiplegia) Occupation: pen, retired \& home duties Diag Code level 3 0-12M: I10 (Essential (primary) hypertension) Diag Code level 4 0-12M: R478 (Other and unspecified speech disturbances) Diag Code level 4 0-12M: 1639 (Cerebral infarction, unspecified) Past radiology 0-12M: MRI Brain
Diag Code level 4 0-12M: G819 (Hemiplegia, unspecified)

Past radiology 0-12M: US Carotid Doppler
isAge60To70

Diag Code level 4 0-12M: I632 (Cerebral infarction due to unspecified occlusion or stenosis of precerebral arteries) isAge70To80 Diag Code level 3 0-12M: 163 (Cerebral infarction)

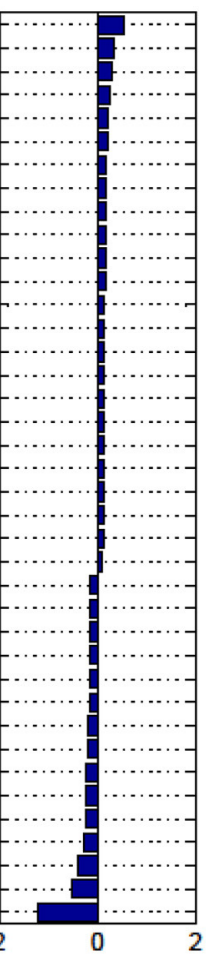

FIGURE A7 | Factors for prediction of Discharge to Nursing Home vs Rehabilitation for the sub-cohort of ischemic stroke patients.

\begin{tabular}{|c|}
\hline 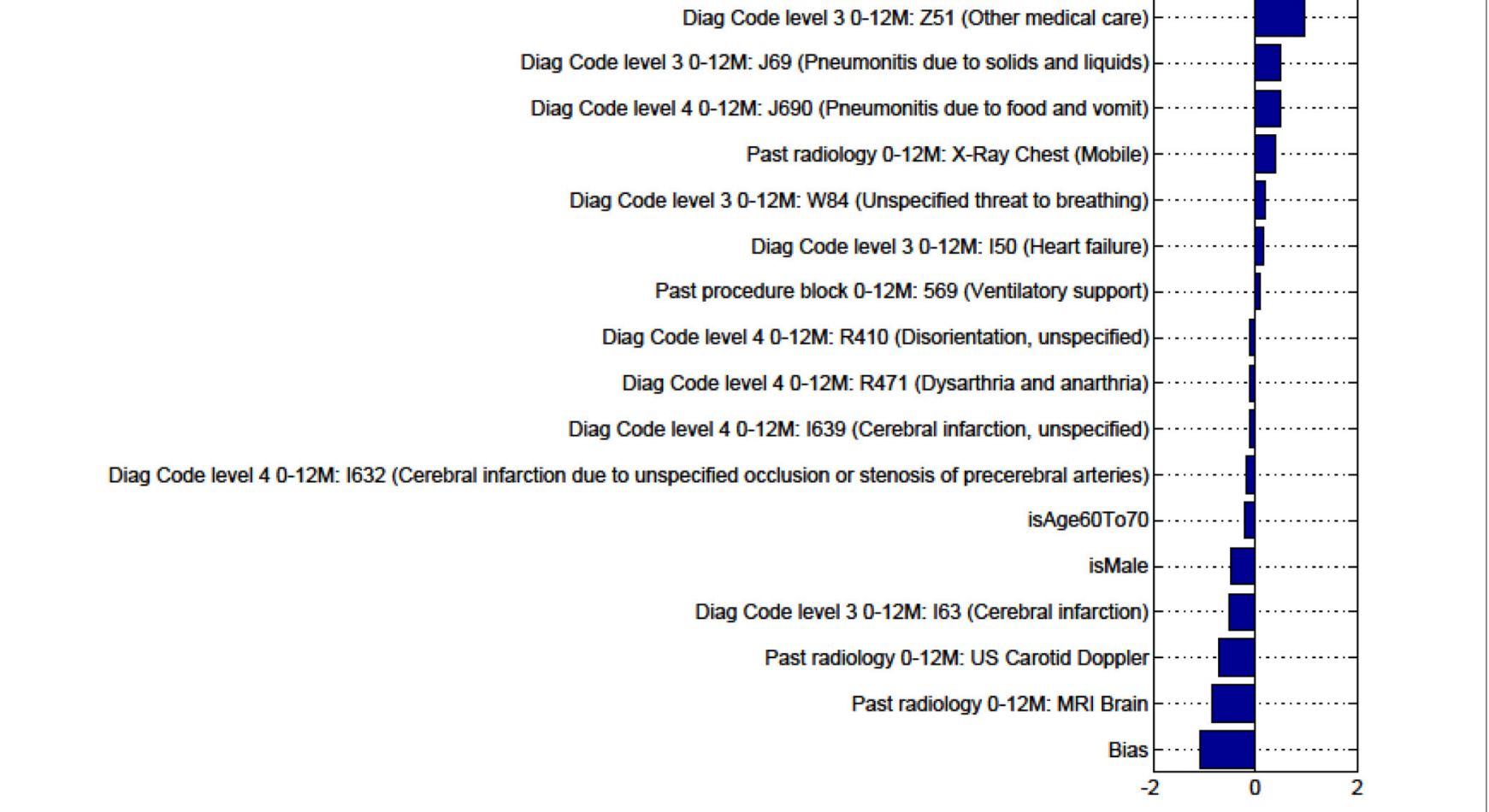 \\
\hline
\end{tabular}




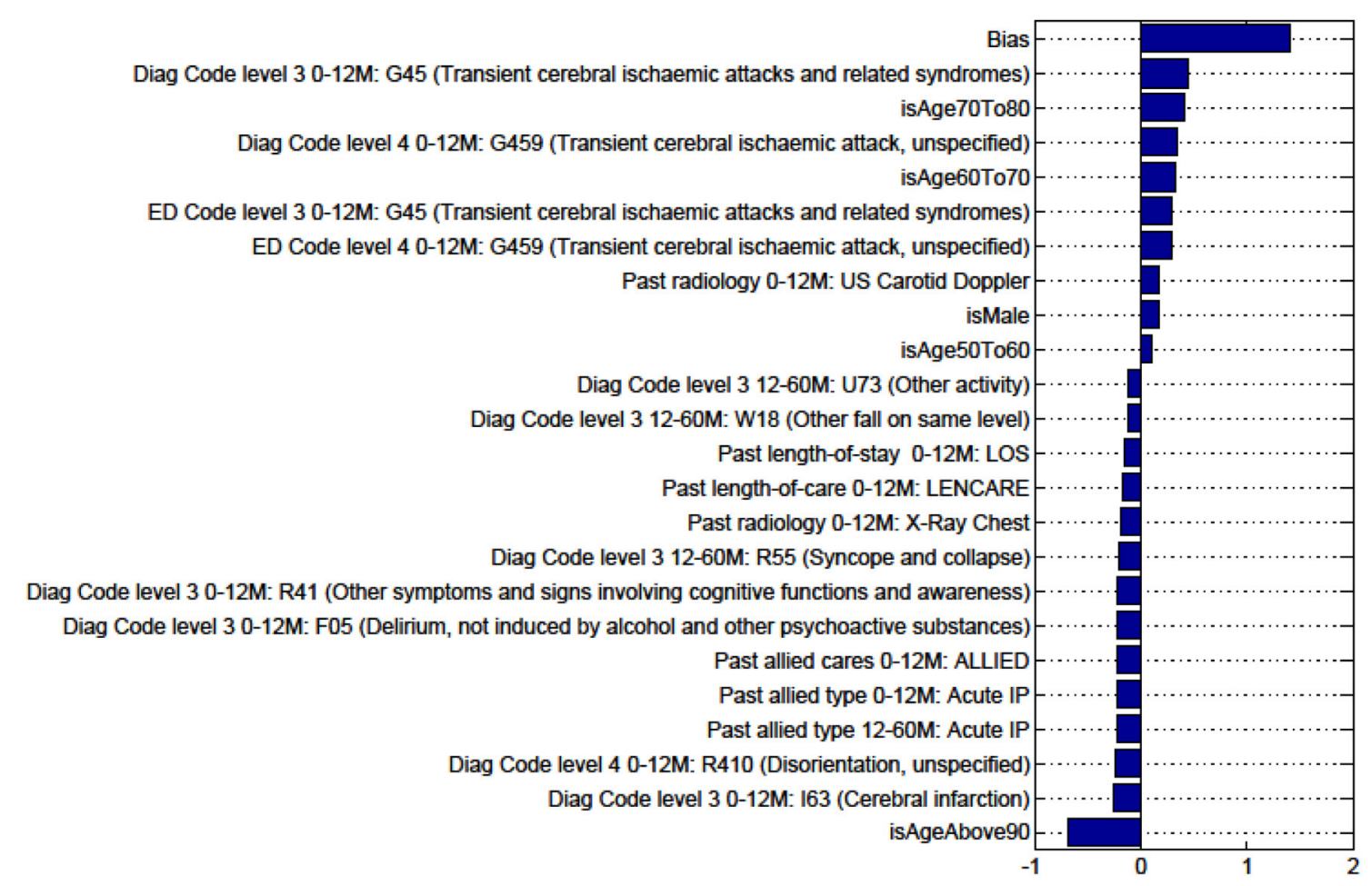

FIGURE A9 | Factors for prediction of Discharge Home vs all Other discharge destinations for the sub-cohort with TIA.

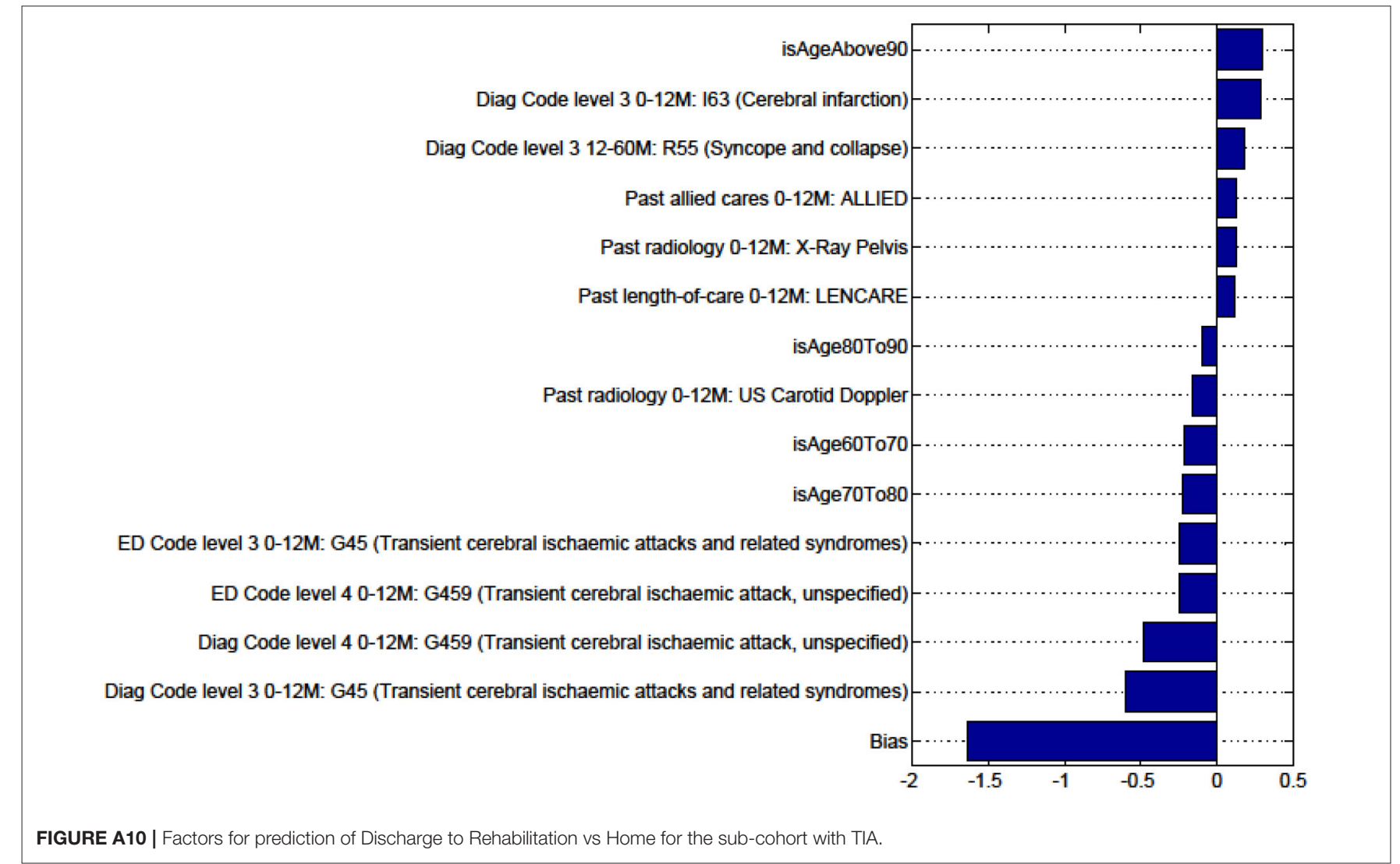

